\title{
High platelet count and high low-density lipoprotein level may be an independent marker of increased arterial stiffness in adult HIV-infected persons
}

Tomasz Mikuła ${ }^{1}$, Michal Peller ${ }^{2}$, Pawel Balsam² ${ }^{2}$ Magdalena M. Suchacz ${ }^{1}$, Mariusz Sapula ${ }^{1}$, Lukasz Koltowski², Renata Glowczynska ${ }^{2}$, Grzegorz Opolski², Krzysztof J. Filipiak², Alicja Wiercinska-Drapalo ${ }^{1}$

${ }^{1}$ Department of Infectious and Tropical Diseases and Hepatology, Medical University of Warsaw, Poland

${ }^{2}$ Chair and Department of Cardiology, Medical University of Warsaw, Poland

\begin{abstract}
Introduction: Cardiovascular disease is an increasing and leading cause of morbidity and mortality in people living with human immunodeficiency virus (PLHIV) infection. Arterial stiffness is a predictor of endothelial dysfunction in subclinical patients. The aim of this study was to assess which of the laboratory parameters performed during the standard PLHIV medical care visit may be associated with increased arterial stiffness.

Material and methods: Thirty HIV-1-infected adult patients (four females and 26 males) were recruited to this study. Endothelial dysfunction and arterial stiffness assessment was performed using the Endo-PAT 2000 device $\left(\right.$ ITAMAR $^{\circledR}$ ). Baseline pressure waveform obtained by peripheral arterial tonometry (PAT) was used to calculate the augmentation index (AI). AI values were corrected for heart rate using an arbitrarily defined reference heart rate of 75 bpm (AI@75).

Results: Our analysis included 30 patients. Men comprised 87\% $(n=26), 16$ patients $(53 \%)$ were on combined antiretroviral therapy (cART), 11 patients (36.7\%) had undetectable HIV viral load, 15 patients (50\%) had active or previous hepatitis $\mathrm{C}$ virus (HCV) or hepatitis B virus (HBV) co-infection, and 16 patients $(53 \%)$ smoked cigarettes. In univariate analysis, only total platelet count and effective cART use influenced arterial stiffness in PLHIV. In multivariate analysis, the variables with confirmed statistical significance were low-density lipoprotein $(\mathrm{LDL})(p=0.040$; slope estimate $=6.30 \mathrm{per} \mathrm{mmol} / \mathrm{l}$ of LDL) and platelet (PLT) count ( $p=0.003$; slope estimate $=0.13$ per $109 / 1$ of platelet count).

Conclusions: We showed that high LDL concentration and high total platelet count may be an independent risk factor of the subclinical atherosclerotic disease in PLHIV. Moreover, we observed a possible negative influence of cART on arterial stiffness.
\end{abstract}

HIV AIDS Rev 2019; 18, 1: 14-18 DOI: https://doi.org/10.5114/hivar.2019.83688

Key words: LDL, total platelet count, cART, arterial stiffness, HIV.

Address for correspondence: Dr. Magdalena Monika Suchacz, Department of Infectious and Tropical Diseases and Hepatology, Medical University of Warsaw, 37 Wolska St., 01-201 Warsaw, Poland, e-mail: m.dabrowska@op.pl
Article history:

Received: 26.02.2019

Received in revised form: 07.03.2019

Accepted: 07.03.2019

Available online: 15.03.2019
International Journal of HIV-Related Problems

HIV \& AIDS

R e v i e w 


\section{Introduction}

Effective combined antiretroviral therapy (cART) has significantly improved the life expectancy in people living with human immunodeficiency virus (PLHIV) infection. Consequently, the age-related co-morbidities have become more common in this population. Cardiovascular disease is an increasing and leading cause of morbidity and mortality in PLHIV, before lung diseases and non-acquired immunodeficiency syndrome (AIDS)-defining cancers [1]. Cardiovascular risk among PLHIV is multifactorial and involves HIV-related and non-HIV-related factors. HIV-related factors include HIV-infection itself and cART-related metabolic disturbances. It has been already shown that some HIV proteins like nef or gp120 may cause progressive endothelial dysfunction [2, 3]. Moreover, HIV infection is linked with chronic immune activation and low-grade inflammation. In antiretroviral (ARV)-naïve HIV-infected patients some lipid disturbances such as increased concentrations of triglycerides (TG) and decreased concentrations of high-density lipoprotein cholesterol (HDL) has been observed [4]. The influence of cART on the increase in cardiovascular risk has been similarly described, especially in older PLHIV patients. ARV drugs, especially protease inhibitors boosted with ritonavir (PIs/r), have a significant impact on serum lipids and increase atherosclerotic lesions $[5,6]$. Non-HIVrelated factors are similar to those seen in the general immunocompetent population and include genetic factors, age, sex, smoking, arterial hypertension, lipid disturbances, diabetes, obesity, sedentary lifestyle, unhealthy diet, and alcohol abuse.

Endothelial dysfunction is an early marker of coronary artery and peripheral arterial disease [7]. Arterial stiffness is a predictor of endothelial dysfunction in subclinical patients. The gold standard for non-invasive endothelial dysfunction measurement is carotid-femoral pulse wave velocity (cf-PWV); however, different evaluation methods such as radial PWV or cardiac magnetic resonance have also been used. Results are varied; some studies show the impact of HIV and cART on increased aortic stiffness while others do not [8]. Endothelial dysfunction may be also measured in peripheral vessels by reactive hyperaemia peripheral arterial tonometry (RH-PAT). It has been shown that persons with endothelial dysfunction in coronary microcirculation also have low peripheral hyperaemic response [9]. Another method of cardiovascular risk assessment is the determination of the augmentation index $(\mathrm{AI})$, which is a non-invasive method of estimating arterial stiffness. AI has been already used as a marker of subclinical atherosclerotic disease in PLHIV [10].

We previously showed that cART had a negative influence on arterial stiffness in PLHIV [11]. However, we did not confirm the significance of the CD4 nadir in progression of endothelial stiffness in this group of patients [12]. The AI determination is not usually available in everyday clinical practice, hence the need for non-invasive, routinely available serum markers of arterial stiffness.
The aim of this study was to assess which of the laboratory parameters performed during the standard PLHIV medical care visit may be associated with increased arterial stiffness.

\section{Material and methods}

Thirty HIV-1-infected adult patients (four females and 26 males; 16 on cART) were recruited to participate in this study. The exclusion criteria were: established cardiovascular disease, treatment with hypotensive, hypolipidaemic, or antidiabetic medication, pregnancy or lactation, ARV treatment for less than six months (but ARV treatment-naïve patients were included), and HIV-associated malignancy. All patients signed a written, informed consent form. The study protocol was reviewed and authorised by the Bioethics Committee of the Medical University of Warsaw.

Each patient underwent history taking and physical examination within seven days prior to evaluation of endothelial function and arterial stiffness. Basic clinical parameters were recorded, including age, body mass index (BMI), smoking (defined as a minimum of five pack-years of smoking), heart rate, blood pressure, and used cART. At the same time, blood samples were taking for laboratory testing. Peripheral blood tests included complete blood count (CDC), evaluation of CD4+ and CD8+ cell count, HIV-1 viral load, creatinine, total bilirubin, C-reactive protein (CRP), total cholesterol, high-density lipoprotein (HDL), low-density lipoprotein (LDL), and triglycerides (TG).

Endothelial dysfunction and arterial stiffness assessment were performed using the Endo-PAT 2000 device $\left(\right.$ ITAMAR $^{\circledR}$ ). The device allows non-invasive evaluation of endothelial function and indirect assessment of vascular stiffness. Its mechanism of action is based on endothelium-dependent arterial response to 5-min brachial artery occlusion. Temporary upper-limb ischaemia results in a subsequent increase in blood flow within the evaluated vascular bed. This response is based on endothelium-dependent vasodilatation in reaction to a local decrease in partial oxygen tension and an increase in potassium ion concentration. In these circumstances, endothelium releases local factors that cause arterial smooth muscle relaxation. Peripheral Arterial Tonometry $\left(\mathrm{PAT}^{\circledR}\right)$ is a patented technology allowing non-invasive measurements of changes in the arterial tone within peripheral vascular beds. The non-invasive Endo-PAT2000 system includes a pair of plethysmographic sensors that impart a uniform pressure field to the distal two thirds of the fingers including their tips. To standardise the results, measurements in all patients were performed in a sitting position after at least $15 \mathrm{~min}$ of rest, allowing stabilisation of the CV system and adjustment to the ambient temperature. In all cases, patients' hands were supported at the heart level. After placing sensors connected to the Endo-PAT2000 device on the index fingers of both hands and a sphygmomanometer cuff on one arm, a baseline measurement was performed. Brachial artery flow was then occluded for 5 min by inflating the cuff. Following this period, 
the pressure was released and further PAT measurements were made. The duration of a single examination was about $15 \mathrm{~min}$. The degree of arterial tone changes in response to ischaemia was expressed as the reactive hyperaemia index (RHI). Normal endothelial function was defined as RHI $>1.67$. This cut off value was suggested by the device manufacturer based on previous studies [13]. A baseline pressure

Table 1. Baseline patients' characteristics - means and standard deviations

\begin{tabular}{|c|c|c|}
\hline Factor & Mean & $\begin{array}{l}\text { Standard } \\
\text { deviation }\end{array}$ \\
\hline Age (years) & 36.5 & 9.5 \\
\hline Al@75 & 1.2 & 14.6 \\
\hline BMI $\left(\mathrm{kg} / \mathrm{m}^{2}\right)$ & 23.6 & 3.8 \\
\hline Systolic blood pressure (mmHg) & 125 & 18 \\
\hline Diastolic blood pressure ( $\mathrm{mmHg}$ ) & 78 & 13 \\
\hline Heart rate (bpm) & 78 & 12 \\
\hline CD4+ cell count (cells/ $\mu$ l) & 353 & 246 \\
\hline CD8+ cell count (cells/ $\mu \mathrm{l})$ & 793 & 403 \\
\hline Haemoglobin level (g/dl] & 14.0 & 1.8 \\
\hline White blood cell count $\left(10^{9} / \mathrm{l}\right)$ & 6.5 & 2.3 \\
\hline Platelet count $\left(10^{9} / \mathrm{l}\right)$ & 195 & 64 \\
\hline Serum creatinine $(\mu \mathrm{mol} / \mathrm{l})$ & 73 & 16 \\
\hline Serum bilirubin $(\mu \mathrm{mol} / \mathrm{l})$ & 18 & 23 \\
\hline C-reactive protein (mg/l) & 15 & 31 \\
\hline Total cholesterol (mmol/l) & 4.6 & 1.1 \\
\hline Low-density lipoprotein (mmol/l) & 2.8 & 0.9 \\
\hline High-density lipoprotein $(\mathrm{mmol} / \mathrm{l})$ & 1.2 & 0.5 \\
\hline Triglycerides (mmol/l) & 1.8 & 0.9 \\
\hline
\end{tabular}

Al@75 - augmentation index at 75 bpm, ARV - on antiretroviral therapy, bpm-beats per minute, $S D$-standard deviation waveform obtained by PAT was used to calculate AI using the height of two systolic waveform peaks. Due to a significant effect of heart rate on AI, AI values were corrected for heart rate using an arbitrarily defined reference heart rate of $75 \mathrm{bpm}$, commonly used in other studies that evaluate arterial stiffness (AI@75) [14].

The $\mathrm{R}$ package was used for all statistical calculations. Appropriate statistical tests: Student's $t$-test, Welch's $t$-test, and Pearson's correlation, were used for each type of variable in the univariate analysis. In the multivariate analysis, a linear model with a stepwise elimination method guided by the $p$ value was used to determine which variables were independently associated with the AI@75. A $p$ value of less than 0.05 was assumed to be statistically significant.

\section{Results}

Our analysis included 30 patients, men comprised $87 \%(n=26), 16$ patients (53\%) were on cART, but only 11 of them had undetectable HIV viral load, 15 patients (50\%) had active or previous hepatitis $\mathrm{C}$ virus (HCV) or hepatitis $\mathrm{B}$ virus (HBV) co-infection, and 16 patients (53\%) smoked cigarettes. Fourteen (87.5\%) ARV-treated subjects obtained two nucleoside reverse transcriptase inhibitors (NRTIs), 11 subjects $(68.7 \%)$ ritonavir-boosted protease inhibitor $(\mathrm{PI} / \mathrm{r})$, five subjects $(31.2 \%)$ non-nucleoside reverse transcriptase inhibitors (NNRTIs), and one subject (6.25\%) integrase inhibitor (II). Patients' baseline characteristics are shown in Table 1.

In univariate analysis, only total platelet count and effective cART use with undetectable HIV viral load influenced the arterial stiffness in PLHIV. Pearson's correlations of AI@75 with qualitative variables are shown in Table 2, and the comparison of AI@75 in different groups with Student's $t$-test and Welch's $t$-test is shown in Table 3.

All variables analysed in univariate analysis were included in multivariate analysis. Slope estimates were included to

Table 2. Pearson's correlations of Al@75 with qualitative variables

\begin{tabular}{|c|c|c|c|}
\hline Variable correlated with AI@75 & $R$ & $R^{2}$ & $p$ \\
\hline Age & 0.26 & 0.068 & 0.163 \\
\hline BMI & -0.14 & 0.020 & 0.459 \\
\hline Systolic blood pressure (mmHg) & -0.06 & 0.004 & 0.717 \\
\hline Diastolic blood pressure (mmHg) & 0.05 & 0.003 & 0.781 \\
\hline Heart rate (bpm) & -0.27 & 0.073 & 0.148 \\
\hline CD4+ cell count $($ cell/ $\mu \mathrm{l})$ & -0.09 & 0.008 & 0.647 \\
\hline CD8+ cell count (cell/ $\mu \mathrm{l})$ & 0.09 & 0.009 & 0.618 \\
\hline Triglycerides $(\mathrm{mmol} / \mathrm{l})$ & 0.15 & 0.022 & 0.431 \\
\hline Total cholesterol $(\mathrm{mmol} / \mathrm{l})$ & -0.06 & 0.004 & 0.732 \\
\hline High-density lipoprotein (mmol/l) & 0.16 & 0.025 & 0.408 \\
\hline Low-density lipoprotein (mmol/l) & 0.10 & 0.011 & 0.569 \\
\hline Platelet count $\left(10^{9} / \mathrm{l}\right)$ & 0.41 & 0.170 & 0.023 \\
\hline
\end{tabular}

Al@75-augmentation index at 75 bpm, BMI - body mass index 
Table 3. Comparison of Al@75 in different groups with Student's $t$-test and Welch's $t$-test

\begin{tabular}{|c|c|c|c|c|c|}
\hline \multirow{2}{*}{ Variable } & \multicolumn{2}{|c|}{ Yes } & \multicolumn{2}{|c|}{ No } & \multirow{2}{*}{$p$} \\
\hline & Mean Al@75 & SD & Mean Al@75 & SD & \\
\hline Male sex & -0.3 & 14.4 & 10.5 & 14.1 & 0.175 \\
\hline Detectable HIV viral load & -2.8 & 11.6 & 8.1 & 17.1 & 0.046 \\
\hline ARV & 6.2 & 16.4 & -4.6 & 10.0 & 0.042 \\
\hline Past or current hepatitis B or C & 2.9 & 14.9 & -0.6 & 14.7 & 0.518 \\
\hline Smoking & 2.7 & 15.2 & -0.6 & 14.3 & 0.536 \\
\hline
\end{tabular}

Al@75 - augmentation index at 75 bpm, ARV - on antiretroviral therapy, detectable HIV viral load - > 40 copies $/ \mathrm{ml}$, SD - standard deviation

show the magnitude of effect on the AI@75 per unit of the dependent variable. The only variables with confirmed statistical significance were LDL $(p=0.040$; slope estimate $=6.30$ per $\mathrm{mmol} / \mathrm{l}$ of LDL) and total platelet count ( $p=0.003$; slope estimate $=0.13$ per $10^{\circ} / 1$ of platelet count).

\section{Discussion}

Our study showed a significant association between total platelet count and arterial stiffness in PLHIV. Sloan et al. previoulsy observed that mean platelet volume (MPV) and platelet count play an important role in cardiovascular risk in the general population [15]. Other studies showed that MPV and neutrophil-to-lymphocyte ratio are independent predictors of intra-hospital mortality in patients with acute myocardial infarction. Moreover, it has been shown that MPV-to-platelet ratio is better than MPV itself in predicting long-term adverse outcomes in ST-elevation myocardial infarction (STEMI) patients after percutaneous coronary intervention [16, 17]. However, several studies underline that MPV should be used with caution for the interpretation of cardiovascular risk, because MPV depends on many factors and should be determined by standardided methods $[18,19]$. In our study, we did not analyse MPV but only total platelet count, in the evaluation of cardiovascular risk in PLHIV. Vinholt et al. showed that total platelet count in the range $301-450 \times 10^{9} / 1$ was associated with higher mortality and risk of future cardiovascular disease in the immunocompetent population [20]. Our study confirmed a positive correlation between platelet count and arterial stiffness in PLHIV. More studies are needed to determine total platelet count value in cardiovascular risk assessment in this vulnerable group of patients.

Many publications confirmed the significance of LDL in the progression of atherosclerotic lesions in arterial vessels and in the higher cardiovascular risk in HIV-infected and non-HIV-infected patients $[21,22]$. In our study, high LDL level was also a significant and independent factor of arterial stiffness in PLHIV. This is compatible with present European guidelines in which LDL level is the main risk factor of cardiovascular disease development. Recommended target levels for LDL are optimally $\leq 2 \mathrm{mmol} / \mathrm{l}(80 \mathrm{mg} / \mathrm{dl})$ and standardly $\leq 3 \mathrm{mmol} / \mathrm{l}(115 \mathrm{mg} / \mathrm{dl})$ [23].
In our study, almost $68 \%$ of the ARV-treated group received PI/r, which may have had a significant influence on the observed serum lipid disturbances. Among them, one patient was on darunavir, two patients on atazanavir, and six on lopinavir; all of them were boosted with ritonavir. Our results are similar to Schillaci et al., who confirmed the impact of PI treatment on aortic stiffness [24]. In our study lower AI@75 values (also with negative results) reflect lower arterial stiffness. Consequently, our patients with detectable HIV viral load and ARV-naive had lower aortic stiffness than successfully ARV-treated subjects, which indirectly confirms the negative cART influence on arterial stiffness. However, we observed no association of AI@75 with cART and an undetectable HIV viral load in multivariate analysis. Thus, the influence of confounding factors is possible on these findings.

One limitation of this study is the fact that only $37 \%$ of ARV-treated patients had undetectable viral load. Moreover, more than half of the patients smoked cigarettes, which may have influenced the obtained results. Finally, we used an indirect method to evaluate arterial stiffness based on AI measurements.

\section{Conclusions}

Our study showed that high LDL concentrations and high total platelet count may be an independent risk factor of subclinical atherosclerotic disease in PLHIV. Moreover, we observed possible negative influence of CART on arterial stiffness. Consequently, we suggest that non-invasive measurement of arterial stiffness should be done in every HIV-infected subject with lipid disorders and/or high platelet count, especially on suppressive cART, to primarily detect and lower cardiovascular risk in this group of patients.

\section{Conflict of interest}

The authors declare no potential conflicts of interest with respect to the research, authorship, and/or publication of this article.

\section{References}

1. Palella FJ Jr, Baker RK, Moorman AC, et al. Mortality in the highly active antiretroviral therapy era: changing causes of death and dise- 
ase in the HIV outpatient study. J Acquir Immune Defic Syndr 2006; 43: 27-34.

2. Wang T, Green LA, Gupta SK, et al. Transfer of intracellular HIV Nef to endothelium causes endothelial dysfunction. PLoS One 2014; 9: e91063.

3. Jiang J, Fu W, Wang X, et al. HIV gp120 induces endothelial dysfunction in tumour necrosis factor- $\alpha$-activated porcine and human endothelial cells. Cardiovasc Res 2010; 87: 366-374.

4. Gowdaiah P, Reddy S, Joseph J. Clinical study of lipid abnormalities in anti-retroviral treatment-naive HIV patients. Int J Adv Med 2015; 2: 365-369.

5. Knobel H, Domingo P, Suarez-Lozano I, et al. Rate of cardiovascular, renal and bone disease and their major risks factors in HIV-infected individuals on antiretroviral therapy in Spain. Enferm Infecc Microbiol Clin 2018; doi: 10.1016/j.eimc.2018.09.015 [Epub ahead of print].

6. Alvi RM, Neilan AM, Tariq N, et al. Protease Inhibitors and Cardiovascular Outcomes in Patients With HIV and Heart Failure. J Am Coll Cardiol 2018; 72: 518-530.

7. Ross R. The pathogenesis of atherosclerosis: a perspective for the 1990s. Nature 1993; 362: 801-809.

8. Leite LH, Cohen A, Boccara F. HIV infection and aortic stiffness. Arch Cardiovasc Dis 2017; 110: 495-502.

9. Bonetti PO, Pumper GM, Higano ST, et al. Noninvasive identification of patients with early coronary atherosclerosis by assessment of digital reactive hyperemia. J Am Coll Cardiol 2004; 44: 2137-2141.

10. Yannoutsos A, Ghosn J, Blacher J. Arterial hemodynamic parameters and cardiovascular risk in HIV patients with controlled infection. Presse Med 2018; 47: 3-10.

11. Balsam P, Mikuła T, Peller M, et al. Evaluation of endothelial function and arterial stiffness in HIV-infected patients: a pilot study. Kardiol Pol 2015; 73: 344-351.

12. Mikuła T, Balsam P, Peller M, et al. The effects of CD4 nadirs on vessel stiffness in HIV patients undergoing antiretroviral therapy. Kardiol Pol 2017; 75: 749-757.

13. Bonetti PO, Pumper GM, Higano ST. Research highlights - editorial review of a noninvasive test for endothelial dysfunction. Nature Clin Practice Cardiovasc Med 2005; 2: 64-65.

14. Vlachopoulos C, Aznaouridis K, Stefanadis C. Prediction of cardiovascular events and all-cuse mortality with arterial stiffness: a systematic review and meta-analysis. J Am Coll Cardiol 2010; 55: 1318-1327.

15. Sloan A, Gona P, Johnson AD. Cardiovascular correlates of platelet count and volume in the Framingham Heart Study. Ann Epidemiol 2015; 25: 492-498.

16. Monteiro Júnior JGM, Torres DOC, da Silva MCFC, et al. Prognostic value of hematological parameters in patients with acute myocardial infarction: Intrahospital outcomes. PLoS One 2018; 13: e0194897.

17. Tian C, Song J, He D, et al. Predictive value of mean platelet volu$\mathrm{me} / \mathrm{platelet}$ count for prognosis in acute myocardial infarction. Int Heart J 2018; 59: 286-292.

18. Noris P, Melazzini F, Balduini CL. New roles for mean platelet volume measurement in the clinical practice? Platelets 2016; 27 607-612.

19. Choi DH, Kang SH, Song H. Mean platelet volume: a potential biomarker of the risk and prognosis of heart disease. Korean J Intern Med 2016; 31: 1009-1017.

20. Vinholt PJ, Hvas AM, Frederiksen H, et al. Platelet count is associated with cardiovascular disease, cancer and mortality: a populationbased cohort study. Thromb Res 2016; 148: 136-142.

21. Lawler PR, Akinkuolie AO, Chu AY, et al. Atherogenic lipoprotein determinants of cardiovascular disease and residual risk among individuals with low low-density lipoprotein cholesterol. J Am Heart Assoc 2017; 6: e005549.
22. Maggi P, Di Biagio A, Rusconi S, et al. Cardiovascular risk and dyslipidemia among persons living with HIV: a review. BMC Infect Dis 2017; 17: 551.

23. http://www.eacsociety.org/guidelines/eacs-guidelines/eacsguidelines.html

24. Schillaci G, De Socio GV, Pirro M, et al. Impact of treatment with protease inhibitors on aortic stiffness in adult patients with human immunodeficiency virus infection. Arterioscler Thromb Vasc Biol 2005; 25: 2381-2385. 